\title{
P I 4- I 4 LB. A low dose of multigene, multiclade HIV DNA given intradermally induces strong and broad immune responses after boosting with heterologous HIV MVA
}

\author{
M Bakari*1, S Aboud ${ }^{1}$, C Nilsson², J Francis'1, D Buma3 , C Moshiro ${ }^{1}$, EA Aris ${ }^{3}$, \\ E Lyamuya ${ }^{1}$, M Janabi $^{3}$, J Mbwana ${ }^{1}$, L Mwanyika4 ${ }^{4}$ R Stout ${ }^{5}$, B Hejdeman ${ }^{6}$,

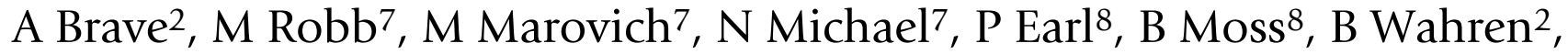 \\ G Biberfeld ${ }^{2}$, K Pallangyo ${ }^{1}$, F Mhalu ${ }^{1}$ and E Sandstrom ${ }^{6}$
}

\begin{abstract}
Address: ${ }^{1}$ Muhimbili University of Health and Allied Sciences (MUHAS), Dar es Salaam, Tanzania, ${ }^{2}$ Swedish Inst for Infectious Disease Control and Karolinska Institute, Stockholm, Sweden, ${ }^{3}$ Muhimbili National Hospital, Dar es Salaam, Tanzania, ${ }^{4}$ Tanzania Police Force, Dar es Salaam, Tanzania, ${ }^{5}$ Bioject, Oregon, USA, ${ }^{6}$ Venhalsan, KI, Sodersjukhuset, Stockholm, Sweden, ${ }^{7}$ Walter Reed Army Institute for Research (WRAIR),

Rockville, MD, USA and ${ }^{8}$ Natl Inst of Allergy \& Infect Dis (NIAID)/Nat Inst of Health (NIH), Bethesda, MD, USA

* Corresponding author
\end{abstract}

from AIDS Vaccine 2009

Paris, France. 19-22 October 2009

Published: 22 October 2009

Retrovirology 2009, 6(Suppl 3):P403 doi:10.1 186/1742-4690-6-S3-P403

This abstract is available from: http://www.retrovirology.com/content/6/S3/P403

(c) 2009 Bakari et al; licensee BioMed Central Ltd.

\section{Background}

A phase I/II HIV vaccine trial among healthy volunteers using a multiclade, multigene HIV-1 DNA prime/MVA boost was conducted in Dar es Salaam, Tanzania.

\section{Methods}

Sixty HIV negative volunteers randomised to 3 groups of 20 , received DNA plasmid vaccine at $1 \mathrm{mg}$ intradermally (i.d.) or $3.8 \mathrm{mg}$ intramuscularly (i.m.) or placebo using the Biojector. DNA plasmids (KI/SMI and Vecura) containing HIV-1 gp160 subtypes A, B, C; rev B; p17/p24 gag A, B and Rtmut B were given $(n=59)$ at months 0,1 and 3. A single boost of $10^{8} \mathrm{pfu}$ of recombinant MVA expressing HIV-1 env, gag, pol of CRF01_AE (NIAID and WRAIR) or placebo was administered i.m. $(n=50)$ at month 9 . Interferon (IFN)-gamma ELISpot responses were measured after stimulation with peptide pools representing HIV-1 p17B, p24A, p55A, gp120A/B, gp120 B, gp41B, gp160E and PolA. Criteria for positive ELISpot responses were $>55$ spots $/ 106$ cells, $>4 \times$ background and $>4 \times$ baseline.

\section{Results}

Two weeks after the DNA injections, 13/19(68\%) i.d. and 9/19(47\%) i.m. vaccinees responded to Gag. Two weeks after the single MVA boost, all vaccine recipients responded: $35(100 \%)$ to Gag and 31(89\%) to Env peptide pools. The i.d. recipients reacted to more peptide pools than i.m. recipients; $\mathrm{p}=0.007$. While there was no significant difference between responders to the peptide pool spanning the Gag protein, there was a higher response to the Env gp160E peptide pool matching the boost, i.d. vs i.m. responders; median $415(\mathrm{n}=17)$ vs median $187(\mathrm{n}=12) \mathrm{p}=0.003$.

\section{Conclusion}

Low dose, intradermal, multigene, multiclade HIV DNA elicited higher and broader immune responses compared to a higher dose given i.m. after a single boost with MVA containing heterologous HIV genes. All these Tanzanian vaccinees responded regardless of DNA priming route. In addition, env immune responses to the matched heterologous boost were higher in the i.d. DNA recipients. 